EPJ Web of Conferences 112,01003 (2016)

DOI: $10.1051 /$ epiconf/201611201003

(C) Owned by the authors, published by EDP Sciences, 2016

\title{
Studies of the nucleon structure in back-to-back SIDIS
}

\author{
H. Avakian ${ }^{1, a}$
}

${ }^{1}$ Thomas Jefferson National Accelerator Facility, 12000 Jefferson Ave. Suite 5, Newport News, VA 23606

\begin{abstract}
The Deep Inelastic Scattering (DIS) proved to be a great tool in testing of the theory of strong interactions, which was a major focus in last decades. Semi-Inclusive DIS (SIDIS), with detection of an additional hadron allowed first studies of 3D structure of the nucleon, moving the main focus from testing the QCD to understanding of strong interactions and quark gluon dynamics to address a number of puzzles accumulated in recent years. Detection of two hadrons in SIDIS, which is even more complicated, provides access to details of quark gluon interactions inaccessible in single-hadron SIDIS, providing a new avenue to study the complex nucleon structure. Large acceptance of the Electron Ion Collider, allowing detection of two hadrons, produced back-to-back in the current and target fragmentation regions, combined with clear separation of two regions, would provide a unique possibility to study the nucleon structure in target fragmentation region, and correlations of target and current fragmentation regions.
\end{abstract}

\section{Introduction}

The quark-gluon dynamics manifests itself in a set of non-perturbative functions describing all possible spin-spin and spin-orbit correlations. Many experiments worldwide are currently trying to pin down various effects related to the nucleon structure through semi-inclusive deep-inelastic scattering (HERMES at DESY [1-4], COMPASS at CERN [5], Jefferson Lab [6-9]) polarized proton-proton collisions (PHENIX, STAR and BRAHMS at RHIC) [10-12], and electron-positron annihilation (Belle and BaBar) [13-16].

Azimuthal distributions of final state particles in in hard scattering processes, in particular, are sensitive to the orbital motion of quarks and play an important role in the study of transverse momentum distributions (TMDs) of quarks in the nucleon. Transverse momentum dependent distributions of partons, encoded in TMDs, and transverse space distributions of partons, encoded in Generalized Parton Distributions (GPDs), have been widely recognized as key objectives of the JLab $12 \mathrm{GeV}$ upgrade and a driving force behind construction of the Electron Ion Collider (EIC). Correlations of the spin of the target or/and the momentum and the spin of quarks, combined with final state interactions define the azimuthal distributions of produced particles.

During the last few years, the first results on transverse single-spin asymmetries (SSAs) have become available. HERMES and COMPASS [3, 5, 17-21] measurements for the first time directly indicated significant azimuthal moments generated both by the Collins and the Sivers effects. Measurements of SSAs at JLab, performed with longitudinally polarized $\mathrm{NH}_{3}$ [22] and transversely polarized ${ }^{3} \mathrm{He}$

\footnotetext{
ae-mail: avakian@jlab.org
} 
[23-26] indicate that spin orbit correlations may be significant for certain combinations of spins of quarks and nucleons and transverse momentum of scattered quarks. Large spin-azimuthal asymmetries have been observed at JLab for longitudinally polarized beam [6] and transversely polarized target [27], consistent with corresponding measurements at HERMES [28] and COMPASS [29], which have been interpreted in terms of higher twist contributions, related to quark-gluon correlations. Recent measurements of multiplicities and double spin asymmetries as a function of the final transverse momentum of pions in SIDIS at JLab [8, 22] suggest that transverse momentum distributions may depend on the polarization of quarks and possibly also on their flavor. Calculations of transverse momentum dependence of TMDs in different models [30-33] and on lattice [34, 35] indicate that the dependence of the transverse momentum distributions on the quark polarization and flavor may be very significant.

Combination of measurements in a large $Q^{2}$ range from HERMES, COMPASS and JLab, extended to EIC would allow studies of evolution effects and control possible higher twist contributions in the measurements of TMD observables in general, and of the Sivers asymmetry in particular. Wide acceptance of CLAS12 detector at Jefferson Lab (JLab) and EIC would allow also checks of Sivers effect in the target fragmentation region, where it is expected to change the sign [36]. Much higher $Q^{2}$ range accessible at JLab12 with CLAS12 and EIC would allow for studies of $Q^{2}$-dependence of different higher twist spin-azimuthal asymmetries, which, apart from providing important information on quark-gluon correlations are needed for understanding of possible corrections from higher twists to leading twist observables. An important process which can provide independent information on twist-3 TMDs is the di-hadron production in SIDIS described by interference functions [37-43]. In fact, the measurement of SSAs with longitudinally polarized target or beam is sensitive in particular to the twist-3 chiral-odd distribution functions $e$ and $h_{L}$, in combination with the chiral-odd interference fragmentation function $H_{1}^{\Varangle}$ [41]. This effect survives after integration over quark transverse momenta and can be analyzed in the framework of collinear factorization. The dihadron production, thus, becomes a unique tool to study the higher twist effects appearing as $\sin \phi$ modulations in target or beam spin dependent azimuthal moments of the SIDIS cross section [44, 45].
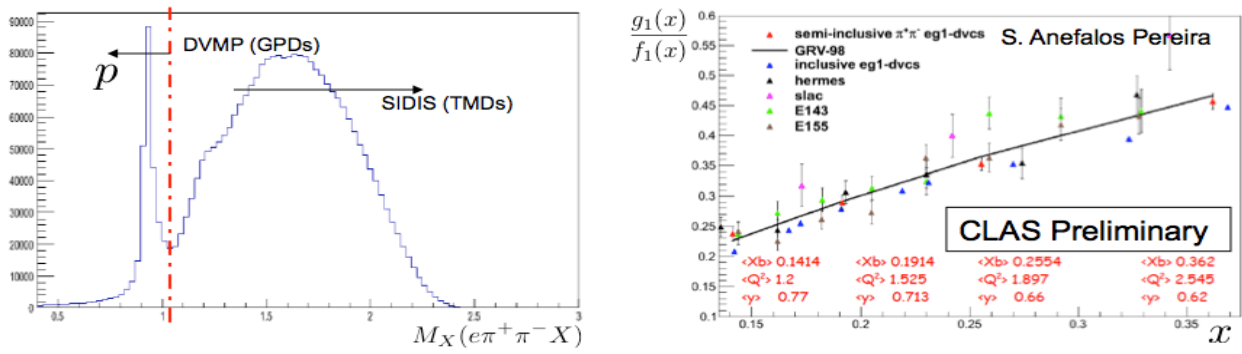

Figure 1. Missing mass of the dihadron system showing the separation of exclusive process (left) and dihadron double spin asymmetry measured at $5.7 \mathrm{GeV}$ in dihadron production, $e \pi^{+} \pi^{-} X$ (red triangles), compared with DIS in different experiments. 


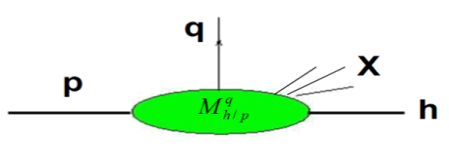

Table 1. TABLE of Leading Twist FFs

\begin{tabular}{|c|c|c|c|}
\hline & $U$ & $L$ & $T$ \\
\hline$U$ & $\hat{u}_{1}$ & $\hat{l}_{1}^{\perp h}$ & $\hat{t}_{1}^{h}, \hat{t}_{1}^{\perp}$ \\
\hline$L$ & $\hat{u}_{1 L}^{\perp h}$ & $\hat{l}_{1 L}$ & $\hat{t}_{1 L}^{h}, \hat{t}_{1 L}^{\perp}$ \\
\hline$T$ & $\hat{u}_{1 T}^{h}, \hat{u}_{1 T}^{\perp}$ & $\hat{l}_{1 T}^{h}, \hat{l}_{1 T}^{\perp}$ & $\hat{t}_{1 T}, \hat{t}_{1 T}^{h h}, \hat{t}_{1 T}^{\perp h}, \hat{t}_{1 T}^{\perp \perp}$ \\
\hline
\end{tabular}

Figure 2. The fracture function defining the conditional probability to produce the hadron $\mathrm{h}$ when a quark $\mathrm{q}$ is struck in a proton target(left) and the full list of leading twist FFs, where U,L,T stand for unpolarized, longitudinally polarized and transversely polarized nucleons (rows) and quarks (columns).

The interference fragmentation function $H_{1}^{\Varangle}$ has been used to obtain information on the transversity parton distribution function [46]. Although, dihadron production in SIDIS requires higher energies and $Q^{2}$, than single hadron SIDIS, measurements of double-spin asymmetries at CLAS are already at $5.7 \mathrm{GeV}$ compatible with simple leading twist predictions for equality of double spin asymmetries in $e X, e \pi^{+} \pi^{-} X$, and $e \pi^{0} X$, assuming the sea quark contributions are negligible at large $\mathrm{x}_{\mathrm{B}}$ and fragmentation functions sum of charged pions are flavor independent. CLAS measurement of the double spin asymmetry from inclusive DIS (also from HERMES and SLAC) are consistent with CLAS measurements of double spin asymmetry in charged pion pair production (see Fig. 1).

Although, the Target Fragmentation Region (TFR) of DIS, when the hadrons are created from the target remnant, carries important information about the spin and flavor structure of the nucleon, it has not been studied systematically in experiments due to lack of theory fundamentals. The main physical question in the TFR is how the diquark-like remnant system after the DIS process dresses itself up to become a full-fledged hadron, i.e., by which mechanism the quark-antiquark pairs restoring color neutrality are produced, and how this process is correlated with the spin of the target or/and the produced particles. In the valence-quark region $(x>0.1)$ accessible at JLab with CLAS detector at $5.7 \mathrm{GeV}$ the polarization transfer from the beam is expected to be significant. High luminosity and high polarization of the electron beam makes CLAS an ideal place for studies of correlations between target and current fragmentation regions.

Recently the leading twist formalism for spin and transverse-momentum dependent fracture functions (FFs) has been developed [47]. The production of two hadrons in polarized SIDIS, with one spinless hadron produced in the current fragmentation region (CFR) and another in the TFR, would provide access to the full set of leading twist FFs [48]. In case of double hadron production process $l(\ell)+$ $N(P) \rightarrow l\left(\ell^{\prime}\right)+h_{1}\left(P_{1}\right)+h_{2}\left(P_{2}\right)+X$, at leading order, the cross-section includes all fracture functions, describing conditional probabilities to produce a hadron of certain type in a target fragmentation for a given flavor of struck quark [47, 48]:

$$
\begin{gathered}
\frac{\mathrm{d} \sigma^{l(\ell, \lambda)+N(P, S) \rightarrow l\left(\ell^{\prime}\right)+h_{1}\left(P_{1}\right)+h_{2}\left(P_{2}\right)+X}}{\mathrm{~d} x \mathrm{~d} y \mathrm{~d} z_{1} \mathrm{~d} \zeta_{2} \mathrm{~d}^{2} \vec{P}_{T 1} \mathrm{~d}^{2} \vec{P}_{T 2} \mathrm{~d} \phi_{S}}= \\
\frac{\alpha^{2} x_{B}}{Q^{4} y}\left[1+(1-y)^{2}\right]\left(\sigma_{U U}+S_{\|} \sigma_{U L}+S_{\perp} \sigma_{U T}+\lambda D_{l l} \sigma_{L U}+\lambda S_{\|} D_{l l} \sigma_{L L}+\lambda S_{\perp} D_{l l} \sigma_{L T}\right),
\end{gathered}
$$

where $D_{l l}(y)=y(2-y) / 1+(1-y)^{2}$. The subscripts in the structure functions $\sigma_{U T, U L, L T}$, specify the beam (first index) and target (second index) polarization ( $U, L, T$ for unpolarized, longitudinally and transversely polarized targets, and $U, L$ for unpolarized and longitudinally polarized beam).

For an unpolarized target expressions there are two contributions $\sigma_{U U}$ and $\sigma_{L U}$, involving convolutions of unpolarized, longitudinally and transversely polarized quark FFs (see Fig. 2) and fragmenta- 
tion functions of unpolarized, $D_{1}$, and transversely polarized quarks, $H_{1}^{\perp}$, [47]:

$$
\begin{gathered}
\sigma_{U U}=F_{0}^{\hat{u} \cdot D_{1}}-D_{n n}\left[\frac{P_{T 1}^{2}}{m_{1} m_{N}} F_{k p 1}^{\hat{t}^{\perp} \cdot H_{1}^{\perp}} \cos \left(2 \phi_{1}\right)+\frac{P_{T 1} P_{T 2}}{m_{1} m_{2}} F_{p 1}^{\hat{t}^{h} \cdot H_{1}^{\perp}} \cos \left(\phi_{1}+\phi_{2}\right)\right. \\
\left.+\left(\frac{P_{T 2}^{2}}{m_{1} m_{N}} F_{k p 2}^{\hat{t}^{\perp} \cdot H_{1}^{\perp}}+\frac{P_{T 2}^{2}}{m_{1} m_{2}} F_{p 2}^{\hat{t}^{h} \cdot H_{1}^{\perp}}\right) \cos \left(2 \phi_{2}\right)\right] . \\
\sigma_{L U}=-\frac{P_{T 1} P_{T 2}}{m_{2} m_{N}} F_{k 1}^{\hat{l}^{\perp h} \cdot D_{1}} \sin \left(\phi_{1}-\phi_{2}\right),
\end{gathered}
$$

The structure functions $F_{\ldots}^{\cdots}$ are specific convolutions [48] of fracture and fragmentation functions depending on $x, z_{1}, \zeta_{2}, P_{1 \perp}^{2}, P_{2 \perp}^{2}, \vec{P}_{1 \perp} \cdot \vec{P}_{2 \perp}$. The hadron 1 produced in the CFR $\left(x_{F 1}>0\right)$ is described by standard scaled variable $z_{1} \simeq P \cdot P_{1} / P \cdot q$, and its transverse momentum $\vec{P}_{T 1}$ (with magnitude $P_{T 1}$ and azimuthal angle $\left.\phi_{1}\right)$ and the hadron $2, h_{2}$ in the TFR $\left(x_{F 2}<0\right)$ is described by similar variables, $\zeta_{2} \simeq$ $E_{2} / E$ and $\vec{P}_{T 2}\left(P_{T 2}\right.$ and $\left.\phi_{2}\right)$, where $x_{F}$ is Feynman variable defining the fraction of the longitudinal momentum of the hadron in the virtual photon-proton center of mass (CM) frame.

In electroproduction, the polarized lepton emits a virtual photon with non-zero longitudinal polarization, which in turn selects preferentially one polarization state of the struck quark. The opposite polarization of a remnant $s \bar{s}$ pair can again be transferred to the final-state $\Lambda$ polarization, with the efficiency extracted from eN collisions. After removing a polarized scattered quark from an unpolarized nucleon, the remnant diquark may combine with an s quark, which could originate from the nucleon sea or from a color string between the diquark and the scattered quark to form a $\Lambda$ hyperon. Significant polarization effects ( 15-20\%) have been predicted in Intrinsic Strangeness Model (ISM) for $\Lambda$ production in the TFR in deep-inelastic scattering [49]. Longitudinal $\Lambda$ polarization transfer coefficient as a function of $x_{F}$ has been already measured with $5.5 \mathrm{GeV}$ electron beam at CLAS (e1f data set). In Fig. 3, the measured asymmetry is shown with the projected results for the future measurements using the CLAS12 detector and $11 \mathrm{GeV}$ beam [50], compared with the ISM predictions [49]. The spin transfer, is proportional to the beam polarization, $P_{B}$, and the depolarization factor, $D(y)$. The meson cloud model [51], due to the pseudoscalar nature of the $N K \Lambda$ coupling, predicts the polarization of final-state $\Lambda$ hyperons to be strongly anticorrelated to that of the nucleon, vanishing for an unpolarized target. The large acceptance of the EIC (see Fig. 4) would provide a unique possibility to study the nucleon structure in the target fragmentation region.

\section{SSA in back-to-back hadron production}

With hadrons detected in the TFR, the beam SSAs appear already at leading order. With two hadrons detected in the final state, structure functions may depend also on the relative azimuthal angle of the two hadrons, generating a long range correlation between hadrons produced in CFR and TFR. The kinematic plane for back-to-back hadron production is shown on Fig. 5. First measurements of single spin asymmetry defined by Eq. 2 in semi-inclusive production of protons and charged pions in coincidence with the scattered electron in hard scattering kinematics $\left(Q^{2}>1 \mathrm{GeV}^{2}, W^{2}>4 \mathrm{GeV}^{2}\right)$ have been performed by the CLAS collaboration using $5.5 \mathrm{GeV}$ and $5.7 \mathrm{GeV}$ longitudinally polarized electron beams scattering off a 5-cm-long liquid-hydrogen target (CLAS e1f and e16 experiments). Target and current fragmentation regions were selected by cuts on the $x_{F}$ variables of protons $\left(x_{F}<0\right)$ and pions $\left(x_{F}>0\right)$, in addition to standard data quality, vertex, acceptance, and fiducial cuts. Based on previous studies we have chosen $0.7>z>0.4$ as the canonical cut on the final state $\pi^{+}$to exclude contamination from exclusive events and decay pions from baryon resonances produced in the target fragmentation region. With a cut on the missing mass of the $e \imath \pi^{+} X$ system, $M_{X}>1.4$ 

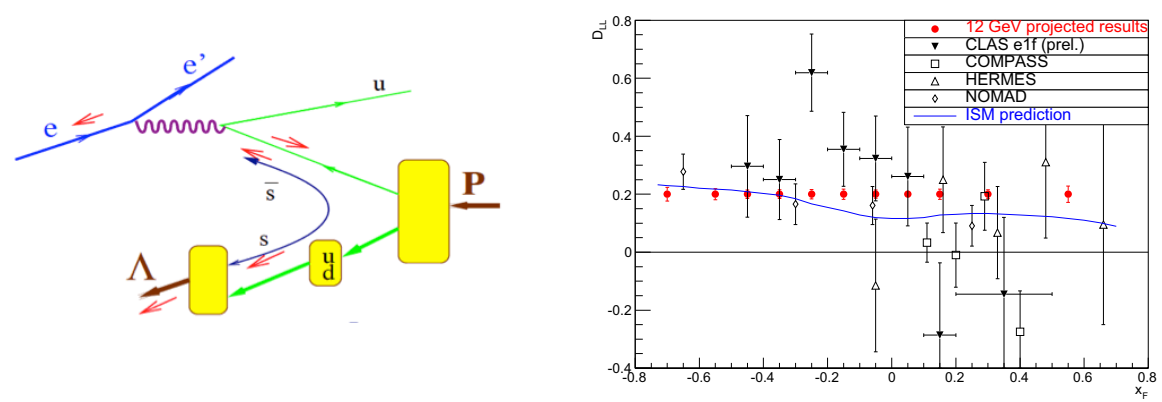

Figure 3. Dominant diagram for $\Lambda$ production in the target fragmentation region due to scattering on a valence $\mathrm{u}$ quark (left) and Longitudinal $\Lambda$ polarization transfer coefficient as a function of $x_{F}$ from CLAS $5.5 \mathrm{GeV}$ elf data set (right).
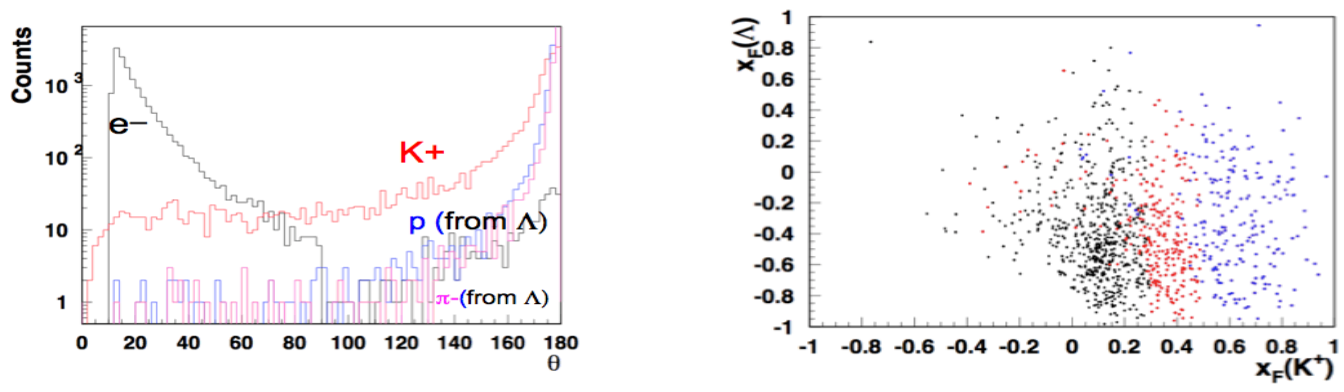

Figure 4. Angular distributions of $e^{\prime} \Lambda K^{+} X$ events for EIC configuration with $5 \mathrm{GeV}$ electrons and $50 \mathrm{GeV}$ protons in the Lab frame (left) and $x_{F}$ distributions for $\Lambda$ s and Kaons for different $z$ fractions of Kaons (from left to wright the lower cut on $z$ increasing from $z>0.1$ to $z>0.5$ ).

we have almost no data at $z>0.7$, but we use this cut as it was determined in Hall $\mathrm{C}$ that strong deviations for the quark-parton model occur at high $z$ [52]. Additional cuts were imposed on the final state $\pi^{+}(0.7>z>0.4)$ to exclude contamination from exclusive events and decay pions from baryon resonances produced in the target fragmentation region. The contamination from target fragmentation, higher twist, or other effects are important for $z<0.3$.

Choosing as independent azimuthal angles $\Delta \phi=\phi_{2}-\phi_{1}$ and $\phi_{2}$, the beam spin asymmetry could be defined as

$$
A_{L U}\left(x, z_{1}, \zeta_{2}, P_{T 1}^{2}, P_{T 2}^{2}, \Delta \phi\right)=\frac{\int \mathrm{d} \phi_{2} \sigma_{L U}}{\int \mathrm{d} \phi_{2} \sigma_{U U}}=\frac{-\frac{P_{T 1} P_{T 2}}{m_{2} m_{N}} F_{k 1}^{\hat{l}^{\perp h} \cdot D_{1}} \sin (\Delta \phi)}{F_{0}^{\hat{u} \cdot D_{1}}} .
$$

The beam spin asymmetry, $A_{L U}^{\sin \Delta \phi}$, has been calculated as a sinusoidal modulation of the difference of azimuthal angles of proton and $\pi^{+}$with respect to the lepton scattering plane, for different electron helicity states. The modulation was extracted for different bins in $x, z$ of the pion and the product of transverse momenta of final state proton and pion with respect to the virtual photon in the CM frame. 

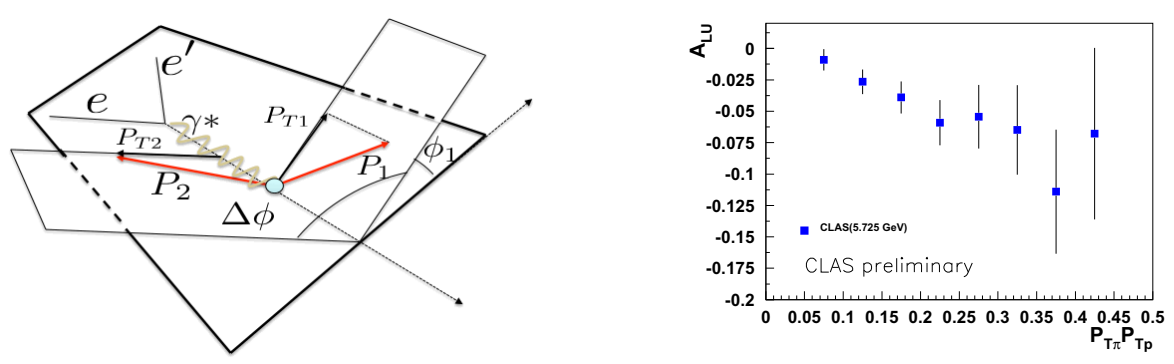

Figure 5. Kinematic plane for back-to-back (b2b) hadron production in SIDIS (left) and measured SSA in backto-back proton and $\pi^{+}$production as a function of the product of their transverse momenta $P_{T 1} P_{T 2}$.

The $P_{T}$-dependence of the SSA shows a trend for asymmetry to increase with increasing transverse momenta of pion and proton $P_{T 1}, P_{T 2}$, (see Fig.5), consistent with expectations from theory. The $x$ dependence of the the $A_{L U}^{\sin \phi}$ is consistent with asymmetry being large in the large- $\mathrm{x}_{\mathrm{B}}$ region, were the valence quark presence is very significant. Similar behavior has been observed also for events with neutral and negative pions in the final state, detected in coincidence with scattered electron and proton in the target fragmentation region.

For quantitative comparison with ongoing measurements as well as projections for future measurements of different $\mathrm{b} 2 \mathrm{~b}$ processes using the CLAS12 and EIC one will need modeling of FFs, which can be modeled using different partonic models used to predict polarization of $\Lambda$ hyperons in the target fragmentation region of DIS, such as the meson cloud model [51] or intrinsic strangeness model for $\Lambda$ production in the target fragmentation region in deep-inelastic scattering [53, 54].

\section{Summary}

In conclusion, kinematic dependences of single spin asymmetries are measured in a wide kinematic range at CLAS with polarized beam and unpolarized hydrogen target. Significant single-spin asymmetries have been observed in back-to-back pion and proton electroproduction for the first time. Measurements of single-spin asymmetries indicate that spin-orbit correlations may play an important role in description of the structure of nucleon in terms of elementary quarks and gluons going beyond the simple collinear partonic representation.

Within the canonical ranges of $x, Q^{2}, z, P_{T}, \phi, M_{X}$, a LO pQCD model of SIDIS, observed asymmetries may be interpreted in a framework using FFs to describe the conditional probabilities of finding partons and hadronization functions describing the fragmentation of the parton to final state hadrons in the current fragmentation. A non-zero $A_{L U}$ in b2b SIDIS, measured for the first time, indicate that spin-orbit correlations between hadrons may be very significant opening a new avenue for studies of the complex nucleon structure in terms of quark and gluon degrees of freedom.

The JLab 12-GeV upgrade will provide the unique combination of wide kinematic coverage, high beam intensity (luminosity), high energy, high polarization, and advanced detection capabilities necessary to study the transverse momentum and spin correlations in di-hadron production in doublepolarized semi-inclusive processes both in the target and current fragmentation regions. The large acceptance of the EIC combined with clear separation of target and current fragmentation regions would provide a qualitatively new tool to study the nucleon structure beyond the traditional current fragmentation. 


\section{References}

[1] A. Airapetian et al. (HERMES), Phys. Rev. Lett. 84, 4047 (2000), hep-ex/9910062

[2] A. Airapetian et al. (HERMES), Phys. Rev. D64, 097101 (2001), hep-ex/0104005

[3] A. Airapetian et al. (HERMES), Phys. Rev. Lett. 94, 012002 (2005), hep-ex/0408013

[4] A. Airapetian et al. (HERMES), Phys. Lett. B648, 164 (2007), hep-ex/0612059

[5] V.Y. Alexakhin et al. (COMPASS), Phys. Rev. Lett. 94, 202002 (2005), hep-ex/0503002

[6] H. Avakian et al. (CLAS), Phys. Rev. D69, 112004 (2004), hep-ex/0301005

[7] H. Avakian, P. Bosted, V. Burkert, L. Elouadrhiri (CLAS), AIP Conf. Proc. 792, 945 (2005), nucl-ex/0509032

[8] H. Mkrtchyan et al., Phys. Lett. B665, 20 (2008), hep-ph/0709. 3020

[9] M. Osipenko et al. (CLAS), Phys. Rev. D80, 032004 (2009), hep-ex/0809. 1153

[10] J. Adams et al. (STAR), Phys. Rev. Lett. 92, 171801 (2004), hep-ex/0310058

[11] M. Chiu (PHENIX), AIP Conf. Proc. 915, 539 (2007), nucl-ex/0701031

[12] I. Arsene et al. (BRAHMS), Phys. Rev. Lett. 101, 042001 (2008), nucl-ex/0801 . 1078

[13] K. Abe et al. (Belle), Phys. Rev. Lett. 96, 232002 (2006), hep-ex/0507063

[14] A. Vossen et al. (BELLE Collaboration), Phys.Rev.Lett. (2011), 1104.2425

[15] I. Garzia et al., Nuovo Cimento 33C, 269 (2010)

[16] I. Garzia (BaBar), Nuovo Cim. C036, 181 (2013)

[17] E.S. Ageev et al. (COMPASS), Nucl. Phys. B765, 31 (2007), hep-ex/0610068

[18] A. Airapetian et al. (HERMES), Phys. Lett. B693, 11 (2010), 1006. 4221

[19] M.G. Alekseev et al. (The COMPASS), Phys. Lett. B692, 240 (2010), 1005.5609

[20] M.G. Alekseev et al., Eur. Phys. J. C70, 39 (2010), 1007.1562

[21] C. Adolph et al. (COMPASS Collaboration), Phys.Lett. B713, 10 (2012), 1202.6150

[22] H. Avakian et al. (CLAS), Phys. Rev. Lett. 105, 262002 (2010), hep-ex/1003 . 4549

[23] X. Qian et al. (Jefferson Lab Hall A), Phys. Rev. Lett. 107, 072003 (2011), 1106.0363

[24] J. Huang et al. (Jefferson Lab Hall A), Phys. Rev. Lett. 108, 052001 (2012), 1108.0489

[25] Y.X. Zhao et al. (Jefferson Lab Hall A), Phys. Rev. C90, 055201 (2014), 1404.7204

[26] Y. Zhang et al. (Jefferson Lab Hall A), Phys. Rev. C90, 055209 (2014), 1312 . 3047

[27] Y.X. Zhao et al. (Jefferson Lab Hall A) (2015), 1502 .01394

[28] A. Airapetian et al. (HERMES), Phys. Lett. B622, 14 (2005), hep-ex/0505042

[29] C. Adolph et al. (COMPASS), Nucl. Phys. B886, 1046 (2014), 1401.6284

[30] Z. Lu, B.Q. Ma, Nucl. Phys. A741, 200 (2004), hep-ph/0406171

[31] M. Anselmino, A. Efremov, A. Kotzinian, B. Parsamyan, Phys. Rev. D74, 074015 (2006), hep-ph/0608048

[32] B. Pasquini, S. Cazzaniga, S. Boffi, Phys. Rev. D78, 034025 (2008), hep-ph/0806. 2298

[33] C. Bourrely, F. Buccella, J. Soffer, Phys.Rev. D83, 074008 (2011), 1008.5322

[34] P. Hagler, B.U. Musch, J.W. Negele, A. Schafer, Europhys. Lett. 88, 61001 (2009), hep-lat/0908.1283

[35] B.U. Musch, P. Hagler, J.W. Negele, A. Schäafer, Phys.Rev. D83, 094507 (2011), 1011. 1213

[36] H.H. Matevosyan, A. Kotzinian, E.C. Aschenauer, H. Avakian, A.W. Thomas, Phys. Rev. D92, 054028 (2015), 1502.02669

[37] R.L. Jaffe, X.m. Jin, J. Tang, Phys. Rev. Lett. 80, 1166 (1998), hep-ph/9709322

[38] A. Bianconi, S. Boffi, R. Jakob, M. Radici, Phys. Rev. D62, 034008 (2000), hep-ph/9907475

[39] M. Radici, R. Jakob, A. Bianconi, Phys. Rev. D65, 074031 (2002), hep-ph/0110252 
[40] A. Bacchetta, M. Radici, Phys. Rev. D67, 094002 (2003), hep-ph/0212300

[41] A. Bacchetta, M. Radici, Phys. Rev. D69, 074026 (2004), hep-ph/0311173

[42] A. Bacchetta, M. Radici, Phys. Rev. D74, 114007 (2006), hep-ph/0608037

[43] F.A. Ceccopieri, M. Radici, A. Bacchetta, Phys. Lett. B650, 81 (2007), hep-ph/0703265

[44] H. Avakian et al. (Jefferson Lab Hall B), PAC38 Proposal (2011)

[45] S. Pisano et al., JLab Experiment E12-06-112B/E12-09-008B (2014), http://www.jlab.org/exp_prog/proposals/14/E12-06-112B_E12-09-008B.pdf

[46] A. Bacchetta, A. Courtoy, M. Radici (2011), 1104. 3855

[47] M. Anselmino, V. Barone, A. Kotzinian, Phys. Lett. B699, 108 (2011), 1102.4214

[48] M. Anselmino, V. Barone, A. Kotzinian, Phys. Lett. B706, 46 (2011), 1109.1132

[49] J.R. Ellis, D. Kharzeev, A. Kotzinian, Z. Phys. C69, 467 (1996), hep-ph/9506280

[50] M. Mirazita et al., JLab Experiment E12-06-112A/E12-09-008A (2014), http://www.jlab.org/exp_prog/proposals/14/E12-06-112A_E12-09-008A.pdf

[51] W. Melnitchouk, A.W. Thomas, Z. Phys. A353, 311 (1995), hep-ph/9508205

[52] T. Navasardyan et al., Phys. Rev. Lett. 98, 022001 (2007), hep-ph/0608214

[53] M.A. Alberg, J.R. Ellis, D. Kharzeev, Phys. Lett. B356, 113 (1995), hep-ph/9503333

[54] J.R. Ellis, M. Karliner, D.E. Kharzeev, M.G. Sapozhnikov, Phys. Lett. B353, 319 (1995), hep-ph/9412334 